\title{
Fine-structure diagnostics of neutral carbon toward HE 0515-4414^
}

\author{
R. Quast, R. Baade, and D. Reimers
}

\author{
Hamburger Sternwarte, Universität Hamburg, Gojenbergsweg 112, 21029 Hamburg, Germany \\ e-mail: [rbaade,dreimers] @hs.uni-hamburg.de
}

Received 30 November 2001 / Accepted 5 March 2002

\begin{abstract}
New high-resolution high signal-to-noise spectra of the $z=1.15$ damped Lyman $\alpha$ (DLA) system toward the quasi-stellar object HE 0515-4414 reveal absorption lines of the multiplets 2 and $3 \mathrm{in} \mathrm{C}$. The resonance lines are seen in two components with total column densities of $\log N=13.79 \pm 0.01$ and $\log N=13.36 \pm 0.01$, respectively. The comparision of theoretical calculations of the relative fine-structure population with the ratios of the observed column densities suggests that the $\mathrm{C}$ I absorbing medium is either very dense or exposed to very intense UV radiation. The upper limit on the local UV energy density is 100 times the galactic UV energy density, while the upper limit on the $\mathrm{H}$ I number density is $110 \mathrm{~cm}^{-3}$. The excitation temperatures of the ground state fine-structure levels of $T=15.7$ and $T=11.1 \mathrm{~K}$, respectively, are consistent with the temperature-redshift relation predicted by the standard Friedmann cosmology. The cosmic microwave background radiation (CMBR) is only a minor source of the observed fine-structure excitation.
\end{abstract}

Key words. cosmic microwave background - intergalactic medium - quasars: absorption lines - quasars: individual: HE 0515-4414

\section{Introduction}

The standard Friedmann cosmology predicts that the temperature of the cosmic microwave background radiation (CMBR) increases linearly with the redshift $z$ as

$T_{\mathrm{CMBR}}(z)=T_{\mathrm{CMBR}}(0)(1+z)$.

The present-day CMBR temperature has been ascertained by the Cosmic Background Explorer FIRAS instrument to be $T_{\mathrm{CMBR}}(0)=2.725 \mathrm{~K}$ (Mather et al. 1999). In recent years several attempts have been made to infer the CMBR temperature at higher redshifts from the relative population of Ci (and CII) fine-structure levels observed in damped Lyman $\alpha$ (DLA) systems (Ge et al. 1997, 2001; Roth \& Bauer 1999; Srianand et al. 2000). The principal problem in this inference is the presence of additional sources of excitation. In particular, collisional excitation and fluorescence induced by the local UV radiation field are competing processes. A recent study of Silva \& Viegas (2002) shows the possibility to assess the physical conditions in QSO absorbers from the observation of finestructure absorption lines.

Send offprint requests to: R. Quast, e-mail: rquast@hs .uni-hamburg.de

* Based on observations made with ESO Telescopes at the La Silla or Paranal Observatories under programme ID 066.A0212 .
This study provides a fine-structure diagnostics of $\mathrm{C}_{\mathrm{I}}$ toward the quasi-stellar object HE 0515-4414 ( $z=1.73$, $B=15.0)$ discovered by the Hamburg/ESO Survey (Reimers et al. 1998). The observed fine-structure absorption lines (and a large number of additional metal lines) are associated with a DLA system at $z=1.15$ (de la Varga et al. 2000). We infer the physical conditions in the DLA system and demonstrate that the CMBR is only a minor source of the observed fine-structure excitation.

\section{Observations}

HE 0515-4414 was observed during ten nights between October 7, 2000 and January 3, 2001, using the UV-Visual Echelle Spectrograph (UVES) installed at the second VLT Unit Telescope (Kueyen). Thirteen exposures were made in the dichroic mode using standard settings for the central wavelenghts of $3460 / 4370 \AA$ in the blue, and 5800/8600 $\AA$ in the red. The CCDs were read out in fast mode without binning. Individual exposure times were 3600 and $4500 \mathrm{~s}$, under photometric to clear sky and seeing conditions ranging from 0.47 to 0.70 arcsec. The slit width was 0.8 arcsec resulting in a spectral resolution of about 55000 in the blue and slightly less in the red.

The raw frames were reduced at Quality Control Garching using the UVES pipeline Data Reduction Software (Ballester et al. 2000). Finally, the individual 
vacuum-barycentric corrected spectra were co-added resulting in an effective signal-to-noise ratio typically better than 100 (up to 130 for the parts of the spectrum considered in this study).

\section{Absorption line analysis}

The observed spectral flux $F$ is the convolution of the instrumental profile $P$ with the product of the background continuum $C$ and the absorption term:

$F(\lambda)=\int P\left(\lambda^{\prime}\right) C\left(\lambda-\lambda^{\prime}\right) \exp \left[-\tau\left(\lambda-\lambda^{\prime}\right)\right] \mathrm{d} \lambda^{\prime}$.

The instrumental profile is approximated by a normalized Gaussian defined by the spectral resolution of the instrument. The background continuum is locally approximated by a linear combination of Legendre polynomials of up to second order. Assuming pure Doppler broadening, the optical depth is modeled by a superposition of Gaussian functions:

$\tau(\lambda)=\sum_{i} g_{i}(\lambda)$,

where

$g_{i}(\lambda)=\frac{e^{2}}{4 \varepsilon_{0} m c} \frac{N_{i} f_{i} \lambda_{i}}{\sqrt{\pi} b_{i}} \exp \left[-\left(c \frac{\lambda /\left(1+z_{i}\right)-\lambda_{i}}{\lambda_{i} b_{i}}\right)^{2}\right]$

with $\lambda_{i}, f_{i}, z_{i}, b_{i}$ and $N_{i}$ denoting the rest wavelength, the oscillator strength, the redshift, the line broadening velocity and the column density of a single line, respectively. Each C I absorption component is modeled by a superposition of Doppler profiles with identical redshifts and widths. Additionally, the particular lines originating on the same fine-structure level are restricted to have identical column densities. This simultaneous fine-structure treatment ensures the physical consistency of our analysis. The weak absorption features between the ${ }^{3} \mathrm{P}_{2}-{ }^{3} \mathrm{P}_{2}$ and ${ }^{3} \mathrm{P}_{1}-{ }^{3} \mathrm{P}_{1}$ lines seen in Fig. 1 are modeled by a superposition of three artificial Doppler profiles.

Our model is defined by 24 strongly correlated parameters: six parameters to model the background continuum, 17 parameters to model the optical depth, and the spectral resolution of the instrument. In order to find the optimal set of parameter values, we minimize the $\chi^{2}$ statistic following an adaptive evolution strategy recently proposed by Hansen \& Ostermeier (2001).

The atomic line data used in this analysis are collected in Table 1.

\section{Results and discussion}

\subsection{Model parameters}

The optimized values and formal confidence limits of the model parameters for both $\mathrm{C}$ I absorption components are collected in Table 2. The total column density of $\mathrm{C}$ I is $\log N=13.79 \pm 0.01$ for the major and $\log N=13.36 \pm$ 0.01 for the minor component. These values are consistent
Table 1. Atomic data for the radiative transitions of the multiplets 2 and 3 in C I. The vacuum wavelengths $\lambda$ are taken from Morton (1991), the oscillator strengths $f$ are adopted from the compilation of Wiese et al. (1996).

\begin{tabular}{lllll}
\hline \hline Mult. & Transition & & $\lambda(\AA)$ & $f\left(10^{-2}\right)$ \\
\hline 2 & $2 \mathrm{p}^{2}-2 \mathrm{p} 3 \mathrm{~s}$ & ${ }^{3} \mathrm{P}_{1}{ }^{3} \mathrm{P}_{2}$ & 1656.2672 & 5.89 \\
& & ${ }^{3} \mathrm{P}_{0}-{ }^{3} \mathrm{P}_{1}$ & 1656.9283 & 13.9 \\
& & ${ }^{3} \mathrm{P}_{2}{ }^{3} \mathrm{P}_{2}$ & 1657.0082 & 10.4 \\
& & ${ }^{3} \mathrm{P}_{1}-{ }^{3} \mathrm{P}_{1}$ & 1657.3792 & 3.56 \\
& & ${ }^{3} \mathrm{P}_{1}-{ }^{3} \mathrm{P}_{0}$ & 1657.9068 & 4.73 \\
3 & ${ }^{3} \mathrm{P}_{2}{ }^{3} \mathrm{P}_{1}$ & 1658.1212 & 3.56 \\
& \multirow{3}{*}{$\mathrm{s}^{2} 2 \mathrm{p}^{2}-2 \mathrm{~s} 2 \mathrm{p}^{3}$} & ${ }^{3} \mathrm{P}_{0}{ }^{3} \mathrm{D}_{1}$ & 1560.3092 & 7.19 \\
& & ${ }^{3} \mathrm{P}_{1}{ }^{3} \mathrm{D}_{2}$ & 1560.6822 & 5.39 \\
& & ${ }^{3} \mathrm{P}_{1}-{ }^{3} \mathrm{D}_{1}$ & 1560.7090 & 1.80 \\
& & ${ }^{3} \mathrm{P}_{2}{ }^{3} \mathrm{D}_{2}$ & 1561.3402 & 1.08 \\
& & ${ }^{3} \mathrm{P}_{2}{ }^{3} \mathrm{D}_{1}$ & 1561.3667 & 0.07 \\
& & ${ }^{3} \mathrm{P}_{2}-{ }^{3} \mathrm{D}_{3}$ & 1561.4384 & 6.03 \\
\hline
\end{tabular}

Table 2. Optimized values and formal 68.3 percent confidence limits of the model parameters for both $\mathrm{C}$ I absorption components. The redshift is accurate to the last digit.

\begin{tabular}{llll}
\hline \hline Level & $z$ & $b\left(\mathrm{~km} \mathrm{~s}^{-1}\right)$ & $\log N\left(\mathrm{~cm}^{-2}\right)$ \\
\hline${ }^{3} \mathrm{P}_{0}$ & 1.150789 & $2.00 \pm 0.03$ & $13.53 \pm 0.01$ \\
${ }^{3} \mathrm{P}_{1}$ & & & $13.35 \pm 0.01$ \\
${ }^{3} \mathrm{P}_{2}$ & & & $12.76 \pm 0.02$ \\
${ }^{3} \mathrm{P}_{0}$ & 1.150853 & $3.53 \pm 0.12$ & $13.21 \pm 0.01$ \\
${ }^{3} \mathrm{P}_{1}$ & & & $12.76 \pm 0.02$ \\
${ }^{3} \mathrm{P}_{2}$ & & & $12.10 \pm 0.07$ \\
\hline
\end{tabular}

with the total column density $\log N=13.90 \pm 0.04$ reported by de la Varga et al. (2000) for the non-separated C I absorption components.

Figure 1 reveals a saturated narrow structure in the ${ }^{3} \mathrm{P}_{0}$ line profiles of the major absorption component. This problem can be tackled indirectly by correcting the apparent optical depth (Savage \& Sembach 1991; Jenkins 1996), but the correction procedure is established for particular synthetic data only and may not be applicable in general. Instead, we tackle the problem of saturated narrow structure directly by testing our analytical procedure with synthetic data similar to the observed spectrum. The tests confirm that our analytical procedure correctly recovers the narrow structure in the ${ }^{3} \mathrm{P}_{0}$ line profiles if the saturation is moderate. Presuming stronger saturation gives rise to observed fine-structure populations being far away from statistical equilibrium.

The optimized value of the spectral resolution of the instrument $R=54600 \pm 800$ matches the spectral resolution of the individual exposures.

\subsection{Physical conditions}

The ground state of the carbon atom consists of the $2 \mathrm{~s}^{2} 2 \mathrm{p}^{2}{ }^{3} \mathrm{P}_{0,1,2}$ fine-structure triplet levels. In DLA systems, the excited levels of the ground state triplet are 

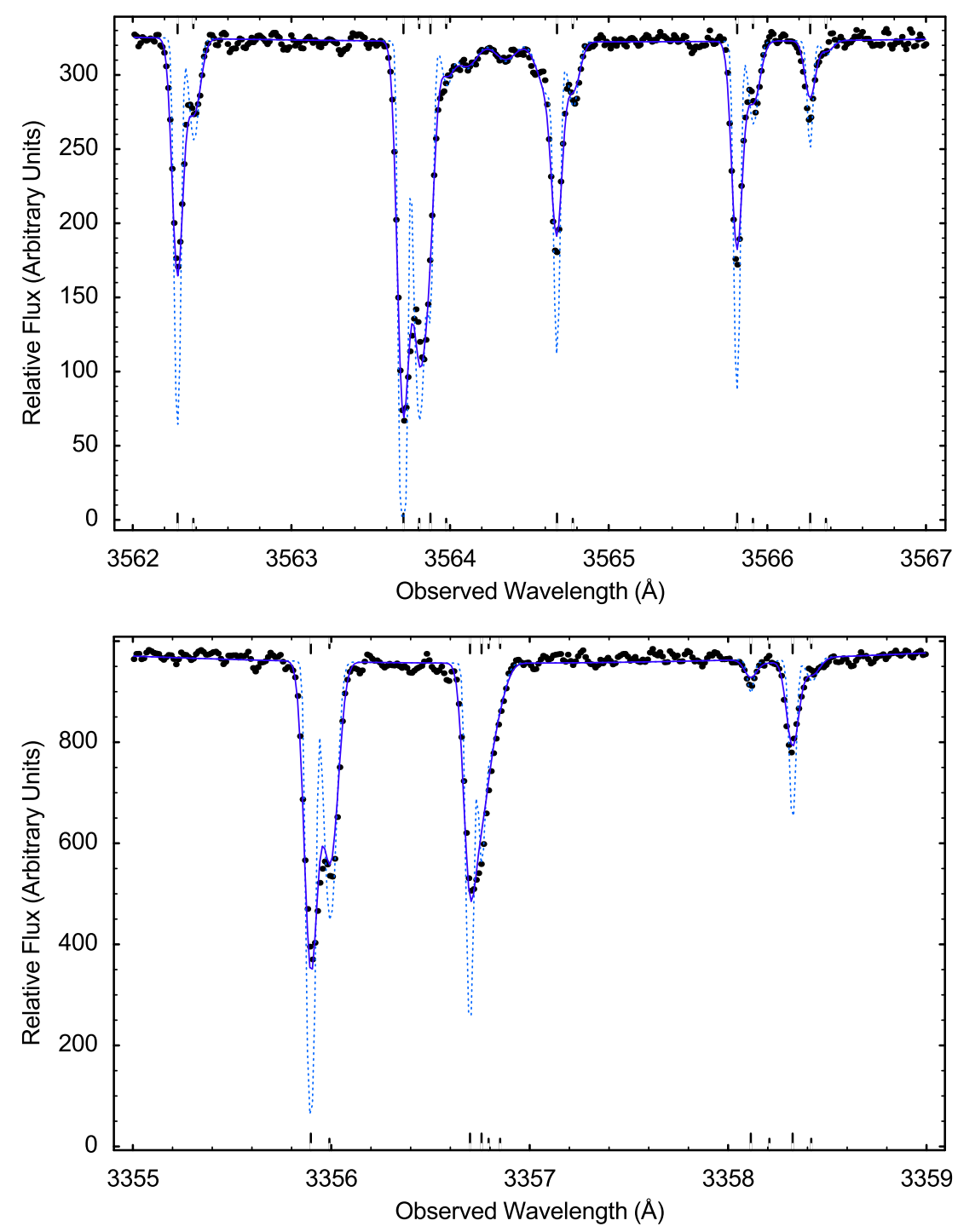

Fig. 1. Parts of the spectrum showing the lines of the multiplets 2 (top panel) and 3 (bottom panel) in $\mathrm{CI}$. The fat dots represent the observed flux. The solid and dashed curves represent our optimized model and its deconvolution, respectively. The long (short) vertical tick marks indicate the major (minor) absorption component in each composite profile. The effective signal-to-noise ratio between the ${ }^{3} \mathrm{P}_{1}-{ }^{3} \mathrm{P}_{1}$ and ${ }^{3} \mathrm{P}_{1}-{ }^{3} \mathrm{P}_{0}$ lines (top panel) is about 130 . The standard error provided with the reduced flux data is about twice the noise.

populated principally by three competing processes: direct photoexcitation by the CMBR, fluorescence induced by the local UV radiation field, and collisional excitation by hydrogen atoms (Bahcall \& Wolf 1968). Given the physical conditions, the relative population of fine-structure levels is determined by solving the system of statistical equilibrium equations. In order to calculate the solution, we use the PopRatio program package recently developed by Silva \& Viegas (2001). The package provides a Fortran 90 source code and an up to date compilation of atomic data for Ci (and Cir, O I, Si II).

The equilibrium equations are established presuming the CMBR temperature follows Eq. (1) and the local UV input equals the scaled generic galactic radiation field. The direct photoexcitation rates by the CMBR at $z=1.15$ are $R_{01}=4.3 \times 10^{-9} \mathrm{~s}^{-1}$ and $R_{02}=2.5 \times 10^{-18} \mathrm{~s}^{-1}$, while fluorescence (the PopRatio package considers 108 UV transitions) induced by the generic galactic UV radiation field (Gondhalekar et al. 1980) yields UV pumping rates of $R_{01}=3.5 \times 10^{-10} \mathrm{~s}^{-1}$ and $R_{02}=2.8 \times 10^{-10} \mathrm{~s}^{-1}$. The H I collision rates at the kinetic temperature $T_{\text {kin }}=100 \mathrm{~K}$ are $q_{01}=3.8 \times 10^{-10} \mathrm{~cm}^{3} \mathrm{~s}^{-1}$ and $q_{02}=2.5 \times 10^{-10} \mathrm{~cm}^{3} \mathrm{~s}^{-1}$.
We point out that the UV pumping rates will exceed the direct photoexcitation rates if the local UV input exceeds the generic galactic radiation field by more than a factor of twelve.

If the absorbing medium is homogenous, the relative population of excited and ground fine-structure levels matches the corresponding column density ratios, $X_{1,2}=$ $N_{1,2} / N_{0}$. Consequently, if the column densities $N_{0,1,2}$ are regarded as independent random observables with density functions $p_{0,1,2}\left(N_{0,1,2}\right), X_{1,2}$ are random observables with cumulative distribution functions

$F\left(X_{1,2}\right)=\int_{0}^{\infty} \int_{0}^{N_{0} X_{1,2}} p_{0}\left(N_{0}\right) p_{1,2}\left(N_{1,2}\right) \mathrm{d} N_{1,2} \mathrm{~d} N_{0}$.

Deriving the cumulative distribution functions with respect to $X_{1,2}$ yields the density functions

$p\left(X_{1,2}\right)=\int_{0}^{\infty} p_{0}\left(N_{0}\right) p_{1,2}\left(N_{0} X_{1,2}\right) N_{0} \mathrm{~d} N_{0}$.

In order to infer the physical conditions in each observed C I absorber, we define $p_{0,1,2}\left(N_{0,1,2}\right)$ by the requirement that $\log N_{0,1,2}$ are normally distributed with mean values 


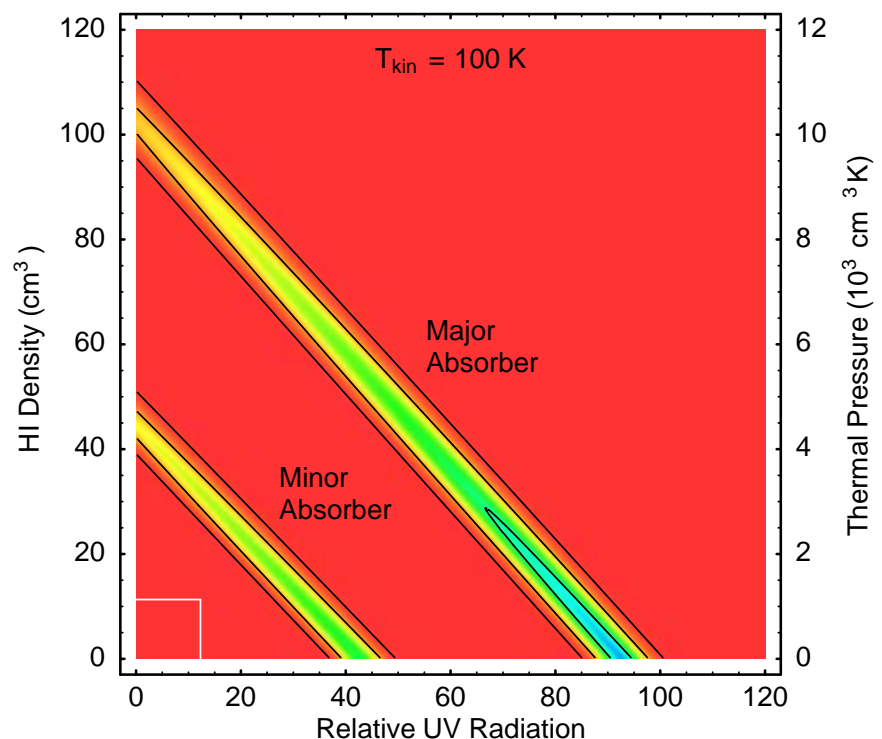

Fig. 2. Probability density of the physical conditions in the DLA system. The contour lines are drawn at $0.61,0.14$, and 0.01 of the maximum and would correspond to the boundaries of the $68.3,95.4$, and 99.7 percent confidence regions if the distributions were normal. The rectangle in the lower left corner marks the physical conditions when the CMBR would be the principal source of the fine-structure excitation.

and standard deviations matching the optimized values collected in Table 2. Then, we calculate the equilibrium population $X_{1,2}$ for a grid of physical conditions and evaluate the joint density function

$p\left(X_{1}, X_{2}\right)=p\left(X_{1}\right) p\left(X_{2}\right)$.

Contour diagrams of the joint density functions obtained in this way are shown in Fig. 2, considering a kinetic temperature of $T_{\text {kin }}=100 \mathrm{~K}$. Figure 2 clearly demonstrates that the CMBR is only a minor source of the observed fine-structure excitation. Considering the 0.01 contour line (i.e. the boundary of the 99.7 percent confidence region if the distribution were normal), the upper limit on the UV energy density in the major (minor) C I absorber is 100 (50) times the galactic UV energy density. The upper limit on the H n number density is $110 \mathrm{~cm}^{-3}\left(50 \mathrm{~cm}^{-3}\right)$. The upper limit on the thermal pressure of $11000 \mathrm{~cm}^{-3} \mathrm{~K}$ $\left(5000 \mathrm{~cm}^{-3} \mathrm{~K}\right)$ exceeds the median pressure in galactic C I absorbers (Jenkins \& Tripp 2001) by a factor of four (two). A similar analysis considering a kinetic temperature of $T_{\text {kin }}=1000 \mathrm{~K}$ (see Fig. 3) yields upper limits of $41 \mathrm{~cm}^{-3}\left(21 \mathrm{~cm}^{-3}\right)$ and $41000 \mathrm{~cm}^{-3} \mathrm{~K}\left(21000 \mathrm{~cm}^{-3} \mathrm{~K}\right)$. If the CMBR is completely excluded from the analysis, the limits increase by less than 20 percent.

If we integrate the joint density function with respect to the H I number density and the relative UV energy density, we obtain the marginal probability density of the kinetic temperature. The marginal density function cannot rule out any kinetic temperature in the range $40 \mathrm{~K} \leq T_{\text {kin }} \leq 1000 \mathrm{~K}$, but indicates that in both C I absorbers a kinetic temperature of about $T_{\text {kin }}=240 \mathrm{~K}$ is the

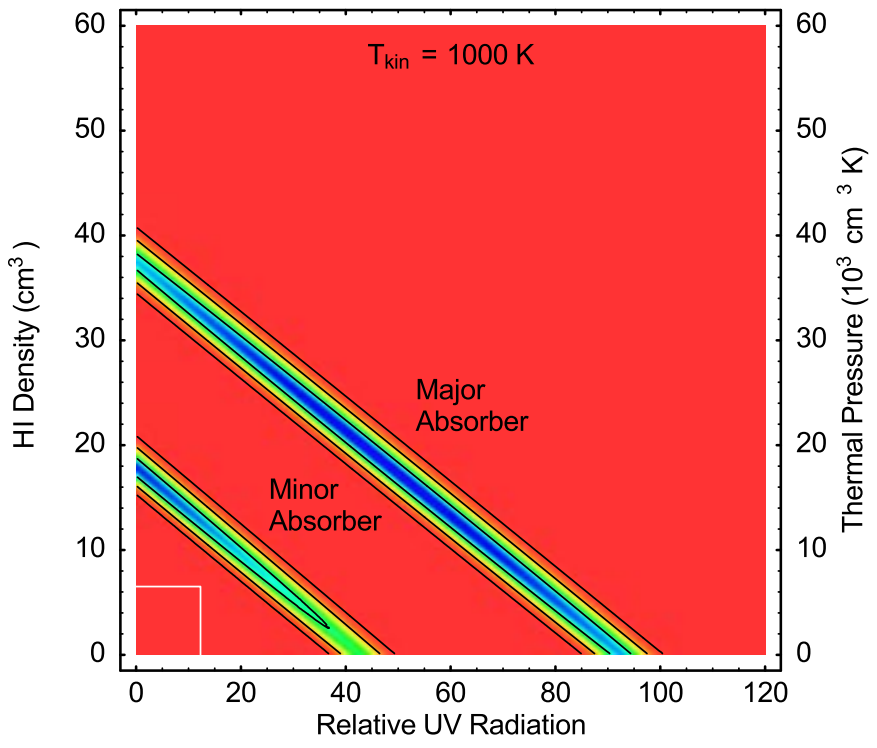

Fig. 3. The same as Fig. 2, but considering a ten times higher kinetic temperature. Note the different scale of the vertical axis.

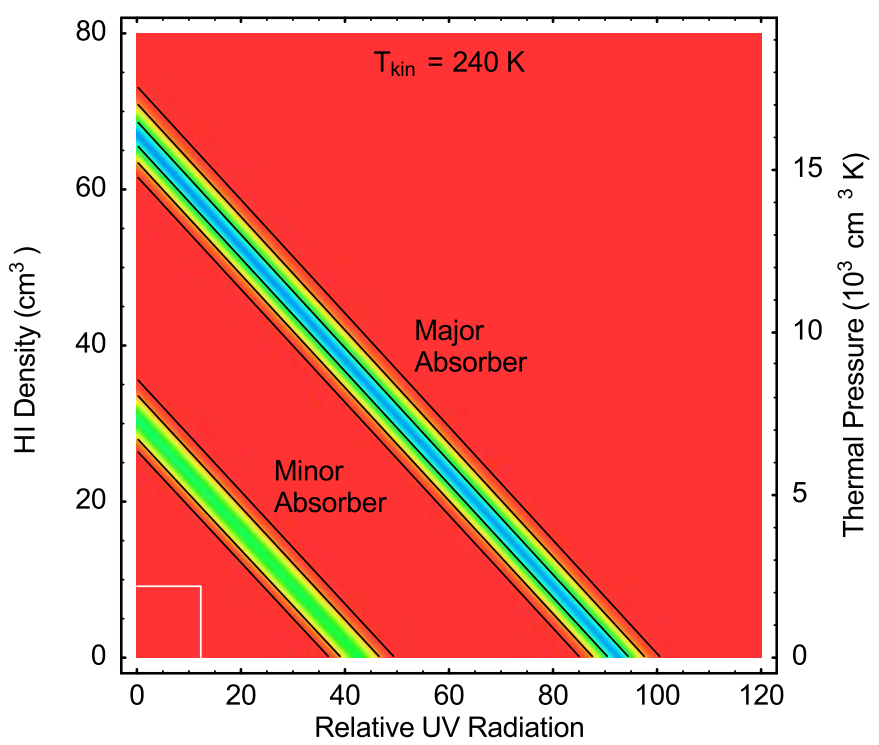

Fig. 4. The same as Figs. 2 and 3, but considering the most probable kinetic temperature. Note the different scale of the vertical axis.

most probable. The corresponding contour plot is shown in Fig. 4.

\subsection{Excitation temperature}

If the absorbing medium is homogenous, the excitation temperature is defined (via the Boltzmann equation) by the ratio of column densities

$\frac{N_{j}}{N_{i}}=\frac{g_{j}}{g_{i}} \exp \left[-\frac{E_{i j}}{k T_{i j}}\right]$.

The energies of the first and second excited ground state fine-structure levels in $\mathrm{C}$ I relative to the ground level are $E_{01}=16.4 \mathrm{~cm}^{-1}$ and $E_{02}=43.4 \mathrm{~cm}^{-1}$. 


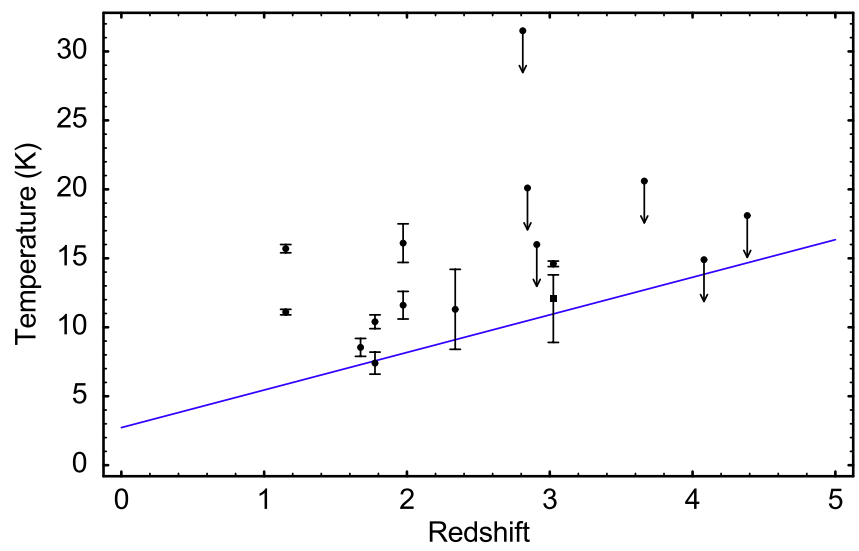

Fig. 5. Fine-structure excitation temperatures derived in this and in previous studies (Songaila et al. 1994; Lu et al. 1996; Ge et al. 1997; Roth \& Bauer 1999; Srianand et al. 2000; Molaro et al. 2002). The square dot marks the CMBR temperature derived by Molaro et al. (2002), the solid line represents the prediction of the standard Friedmann cosmology.

Table 3. Excitation temperatures of the ground state finestructure levels in $\mathrm{C}$. The indices denote the $J$ quantum numbers of the levels considered.

\begin{tabular}{llll}
\hline \hline Absorber & $T_{01}(\mathrm{~K})$ & $T_{02}(\mathrm{~K})$ & $T_{12}(\mathrm{~K})$ \\
\hline Major & $15.7 \pm 0.3$ & $18.5 \pm 0.3$ & $20.9 \pm 0.5$ \\
Minor & $11.1 \pm 0.2$ & $15.0 \pm 0.6$ & $19.2 \pm 1.6$ \\
\hline
\end{tabular}

Now considering the optimized column density values listed in Table 2, we obtain three different excitation temperatures for each $\mathrm{C}$ I absorber (see Table 3 ). These excitation temperatures are considerably higher than the CMBR temperature predicted by Eq. (1), $T_{\mathrm{CMBR}}(1.15)=5.9 \mathrm{~K}$. The magnitude and diversity of the excitation temperatures reveals that the CMBR is not the principal source of the fine-structure excitation. Moreover, the excitation temperatures in the minor absorber are systematically lower, indicating completely different physical conditions.

The fine-structure excitation temperatures derived in this and in previous studies considering higher redshifts are compared in Fig. 5.

\section{Conclusions}

Our theoretical calculations of the relative population of the ground state fine-structure levels in C I clearly demonstrate that the CMBR is only a minor source of the observed fine-structure excitation. The ratios of the observed column densities suggest that the C I absorbing medium is either very dense or exposed to very intense UV radiation. The upper limit on the local UV energy density is 100 times the galactic UV energy density, while the upper limit on the $\mathrm{HI}$ number density is $110 \mathrm{~cm}^{-3}$. Whether flourescence induced by the local UV radiation field or collisional excitation by hydrogen atoms is the more important process cannot be concluded yet.

We also observe absorption lines of molecular hydrogen associated with the DLA system. The observed rotational population is strongly inversed, indicating not only collisonal but also radiative excitation. Therefore, the kinetic temperature of the absorbing medium can only be determined in a multilevel population analysis. This analysis is in progress and will possibly unravel the physical processes giving rise to the observed fine-structure population.

Acknowledgements. We kindly acknowledge A. I. Silva and S. M. Viegas for providing their PopRatio program package for the public. We also thank N. Hansen for sending us a manuscript and for providing us with helpful detailed information about his evolution strategy. This research has been supported by the Verbundforschung of the BMBF/DLR under Grant No. 50 OR 99111.

\section{References}

Bahcall, J. N., \& Wolf, R. A. 1968, ApJ, 152, 701

Ballester, P., Modigliani, A., Boitquin, O., et al. 2000, ESO Mess., 101, 31

de la Varga, A., Reimers, D., Tytler, D., Barlow, T., \& Burles, S. 2000, A\&A, 363, 69

Ge, J., Bechtold, J., \& Black, J. H. 1997, ApJ, 474, 67

Ge, J., Bechtold, J., \& Kulkarni, V. P. 2001, ApJ, 547, L1

Gondhalekar, P. M., Phillips, A. P., \& Wilson, R. 1980, A\&A, 85,272

Hansen, N., \& Ostermeier, A. 2001, Evol. Comput., 9, 159

Jenkins, E. B. 1996, ApJ, 471, 292

Jenkins, E. B., \& Tripp, T. M. 2001, ApJS, 137, 297

Lu, L., Sargent, W. L. W., Barlow, T. A., Churchill, C. W., \& Vogt, S. S. 1996, ApJS, 107, 475

Mather, J. C., Fixsen, D. J., Shafer, R. A., Mosier, C., \& Wilkinson, D. T. 1999, ApJ, 512, 511

Molaro, P., Levshakov, S. A., Dessauges-Zavadsky, M., \& D'Odorico, S. 2002, A\&A, 381, L64

Morton, D. C. 1991, ApJS, 77, 119

Reimers, D., Hagen, H.-J., Rodriguez-Pascual, P., \& Wisotzki, L. 1998, A\&A, 334, 96

Roth, K. C., \& Bauer, J. M. 1999, ApJ, 515, L57

Savage, B. D., \& Sembach, K. A. 1991, ApJ, 379, 245

Silva, A. I., \& Viegas, S. M. 2001, Comput. Phys. Commun., 136,319

Silva, A. I., \& Viegas, S. M. 2002, MNRAS, 329, 135

Songaila, A., Cowie, L. L., Hogan, C. J., \& Rugers, M. 1994, Nature, 368, 599

Srianand, R., Petijean, P., \& Ledoux, C. 2000, Nature, 408, 931

Wiese, W. L., Fuhr, J. R., \& Deters, T. M. 1996, J. Phys. Chem. Ref. Data, Mono. 7 